\title{
Community Acquired Methicillin Resistant Staphylococcus aureus (CA-MRSA) among Skin and Soft Tissue Infections
}

\author{
Neelaveni Duraisamy, Nirmala Devi Somasundram* and Vidhyarani Rangasamy \\ Department of Microbiology, Government Mohan Kumaramangalam Medical College, \\ Salem, TamilNadu, India \\ *Corresponding author
}

\section{A B S T R A C T}

Staphylococcus aureus is one of the most important causes of skin and soft tissue infection and also causes serious fatal systemic disease. $S$. aureus is a commensal

Keywords

Community

Acquired

Methicillin

Resistant

Staphylococcus aureus (CA-MRSA)

Article Info

Accepted:

08 January 2020

Available Online:

10 February 2020 in human body and shows a high rate of carrier state in the general population. The emergence and dissemination of Methicillin-resistant Staphylococcus aureus (MRSA) is a global concern in both health care and community settings now a days. To detect the incidence of Community acquired MRSA (CAMRSA infections) in skin and soft tissue infection. To determine the antimicrobial susceptibility pattern of CA-MRSA isolates. Under standard guidelines pus swabs collected and processed. Under CLSI(2106) guidelines, Screening \& phenotyping confirmation of Methicillin resistance was detected by Cefoxitin disc diffusion method which predicts the results for mecA-mediated Oxacillin resistance. Out of total (81) Staphylococcus aureus isolate 24(29.6\%) were identified and confirmed phenotypically as MRSA by using Cefoxitin $(30 \mu \mathrm{g})$ disc diffusion method. Since the emergence and dissemination of Methicillin-resistant Staphylococcus aureus (MRSA) is a global concern in both health care and community settings now a days, early detection of CA-MRSA will be useful for effective and appropriate antibiotic therapy.

\section{Introduction}

Staphylococcus aureus is one of the most important cause of skin and soft tissue infection and also causes serious fatal systemic disease. S. aureus is a commensal in human body and shows a high rate of carrier state in the general population (anterior nares $35 \%$, perineum $20 \%$, axillae $5 \%-10 \%$, and toe webs $5 \%-10 \%)[1]$. Changes in the bacterial surface receptors is the factor that is responsible for staphylococcal resistance against beta-lactamase resistant penicillins such as methicillin and cloxacillin.[2].

The emergence and dissemination of Methicillin-resistant Staphylococcus aureus (MRSA) is a global concern in both health care and community settings now a days.[3] Patients colonized with MRSA have an increased risk for developing infection compared with noncolonized patients and it 
can be easily transmitted to inpatients through health workers and patient attenders.

MRSA was initially recognized as a purely health care associated pathogen. However, its epidemiology is now changing, and it has been increasingly found in healthy individuals without conventional risk factors for MRSA acquisition[4].

Community-Associated Methicillin- Resistant Staphylococcus aureus (CA-MRSA) defined as MRSA strains acquired in the community and in people with no history of risk factors like hospitalization or surgery, permanent indwelling catheters or percutaneous medical device, residence in a long-term care facility, Dialysis, MRSA culture $<48$ hours after hospital admission. They are genetically distinct and thought to have evolved separately from the Healthcare-Associated strain (HA-MRSA). Currently, CA-MRSA is the predominant strain in both community and hospital settings and spread by close skin to skin contact with a person with MRSA infection or colonization or by coming into direct contact with a surface or item contaminated with MRSA.

They are most commonly implicated in skin and soft tissue infection like abscesses or cellulitis, impetigo, folliculitis or furunculosis and less commonly in invasive and severe infections like necrotizing pneumonia, empyema, sepsis and osteomyelitis.

The main objectives of this study to detect the incidence of Community acquired MRSA (CA-MRSA infections) in skin and soft tissue infections. And to determine the antimicrobial susceptibility pattern of CA-MRSA isolates. Also to monitor outbreak.

\section{Materials and Methods}

Type of study: Prospective study.
Place of study: Govt. Mohan Kumaramangalam Medical College Hospital, Salem.

Study Population: Patients attending Medical and Surgical OP

Study duration: 2 months

Nature of sample: Pus and wound (pus) swabs Sample size: 200 samples

\section{Sample collection}

After obtaining ethical committee approval and patient consent, 200 samples were collected from patients who attended Medical and Surgical outpatient department with history suggestive of skin and soft tissue infections (abscess, furuncle and pyoderma). Under aseptic precautions pus and wound swabs were collected and transported to the laboratory immediately. For wound swabs two swabs were collected with adequate material taken from the depth of the wound to avoid contamination of skin flora

\section{Methods}

After receiving the samples, Gram staining was done for all samples. Then the samples were inoculated into Nutrient and 5\% Blood agar and incubated at $37^{\circ} \mathrm{c}$ for $24-48 \mathrm{hrs}$. Isolated pure colonies with morphology suggestive of Gram positive cocci were identified and the isolates were confirmed \& speciated according to standard protocols by Gram staining and various biochemical reactions like Catalase, Slide coagulase, Tube coagulase, and Mannitol salt agar. The isolates were tested for Antimicrobial susceptibility testing by using Kirby - Bauer Disk diffusion method according to the CLSI guidelines with commercially available antimicrobial discs (Hi-Media) on Mueller Hinton agar plates. The Staphylococcus aureus ATCC 25923 strain was used as control strain. The panel of antibiotic discswere used for antibiotic sensitivity 
testing are Amoxyclav (30/10 mcg), Erythromycin (15mcg), Cefotaxime (30 mcg), Gentamicin (10mcg), Amikacin (30 mcg), Ciprofloxacin (5mcg), Doxycycline (10mcg), Cefaperazone/ Sulbactum (75/10mcg), and Vancomycin (30mcg). The colonies of Gram Negative bacilli were identified with IMViC tests.

\section{Phenotypic detection of MRSA}

For screening \& confirmation of Methicillin resistance (MRSA isolates) Cefoxitin $(30 \mu \mathrm{g})$ disc was used since the Clinical and Laboratory Standards Institute (CLSI) guidelines (2016) had recommended Cefoxitin disc diffusion method for the detection of MRSA which predicts results for mecA-mediated Oxacillin resistance. This was performed by using a $30 \mu \mathrm{g}$ Cefoxitin disc and an inhibition zone diameter of $\leq 21 \mathrm{~mm}$ is reported as Methicillin resistant and $\geq 22 \mathrm{~mm}$ is considered as Methicillin sensitive. For quality control Staphylococcus aureus ATCC 25923 strain (Methicillin sensitive -MSSA) was used as a negative control and Staphylococcus aureus ATCC 43300 strain (Methicillin Resistant -MRSA) was used as a positive control.

\section{Results and Discussion}

Out of 200 patients $123(61.5 \%)$ male and $77(38.5 \%)$ female patients had skin and soft tissue infection. Among 200 samples
$182(91 \%)$ were culture positive which includes 112(61.5\%) male and 70(38.5\%) female patients. Out of the 182 culture positives 107(58.8\%) were Gram Positive Cocci and 75 (41.2\%) were Gram Negative Bacilli. Among the 182 isolates, Staphylococcus aureus 81(44.5\%) was the predominant isolate, followed by Klebsiella spp 34(18.7\%), Coagulase negative Staphylococci 26(14.3\%), Escherichia coli 19(10.4\%), Pseudomonas aeruginosa $13(7.1 \%)$ and the least was Proteus spp 9(4.9\%) [Chart 1].

The Antibiotic susceptibility pattern of Gram positive cocci showed $100 \%$ sensitivity to Vancomycin and Linezolid, Amikacin $(86.9 \%)$, Gentamicin (81.3\%), Ciprofloxacin (69.1\%), Cotrimoxazole (68.2\%), Doxycycline (71.9\%), Amox/clav (55.5\%), Cefotaxime (79.4\%), Cefoperazone/ Sulbactum (84.1\%), Chart -2].

Among 81 isolates of Staphylococcus aureus $24(29.6 \%)$ isolates were identified and confirmed phenotypically as MRSA by using Cefoxitin $(30 \mu \mathrm{g})$ disc diffusion method. In a study done by Kunsang Bhutia[5], out of 119 isolates $46(38.7 \%)$ were found to be MRSA which revealed the significant prevalence of MRSA in skin and soft tissue infections. Out of 26 Coagulase negative Staphylococci $7(26.9 \%)$ were found to be Methicillin resistant [Table : 1 \& Chart - 3].

Table.1 Prevalence of MRSA isolates among Staphylococcus aureus

\begin{tabular}{|l|l|c|c|}
\hline S.no & Name of the isolate & MRSA (\%) & MSSA (\%) \\
\hline 1. & Staph aureus $(\mathrm{n}=81)$ & $24(29.6 \%)$ & 57 \\
\hline $\mathbf{2}$ & CONS $(\mathrm{n}=26)$ & $7(26.9 \%)$ & 19 \\
\hline
\end{tabular}




\section{Chart.1}

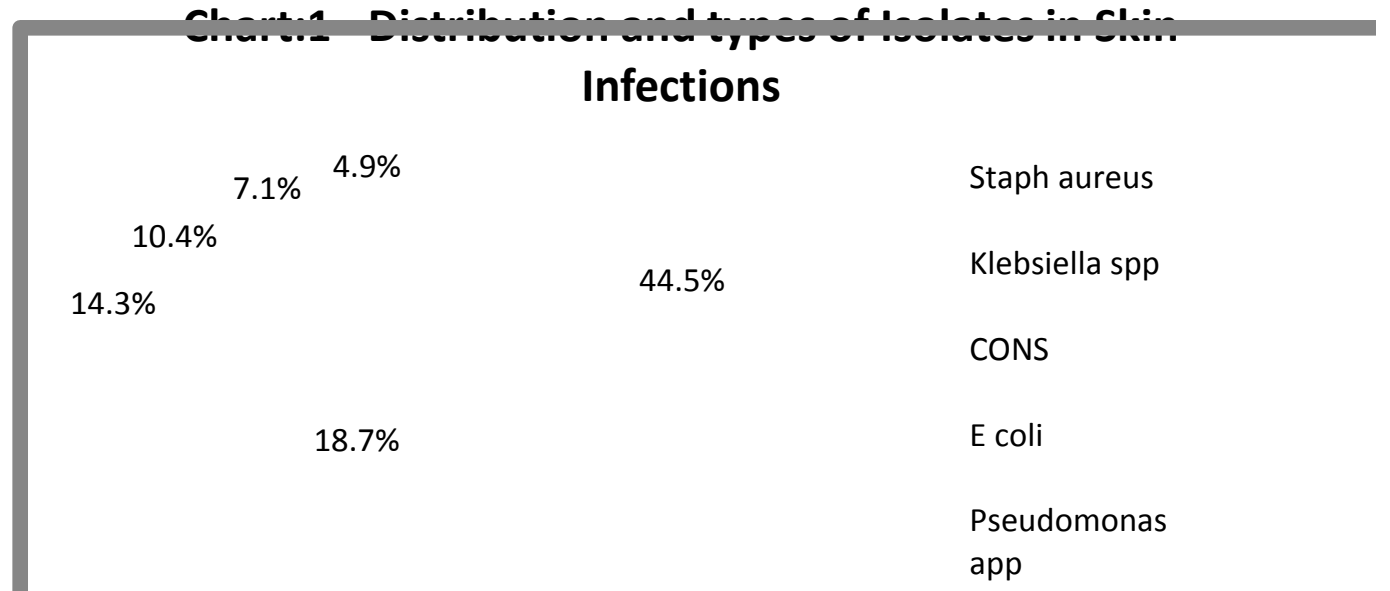

Chart.2 Antibiotic susceptibility pattern for bacterial isolates in skin infection

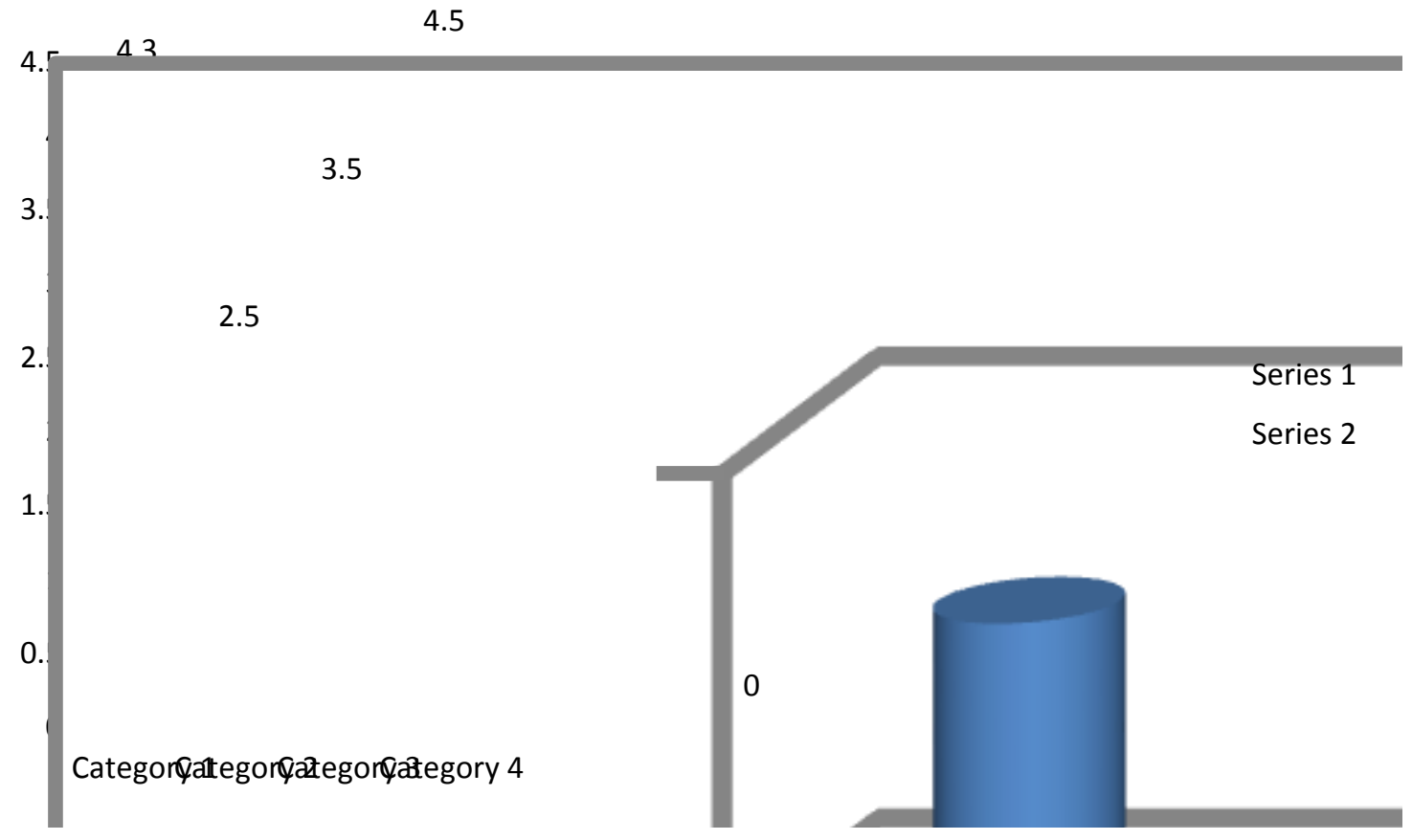


Chart.3 Prevalence of MRSA isolates

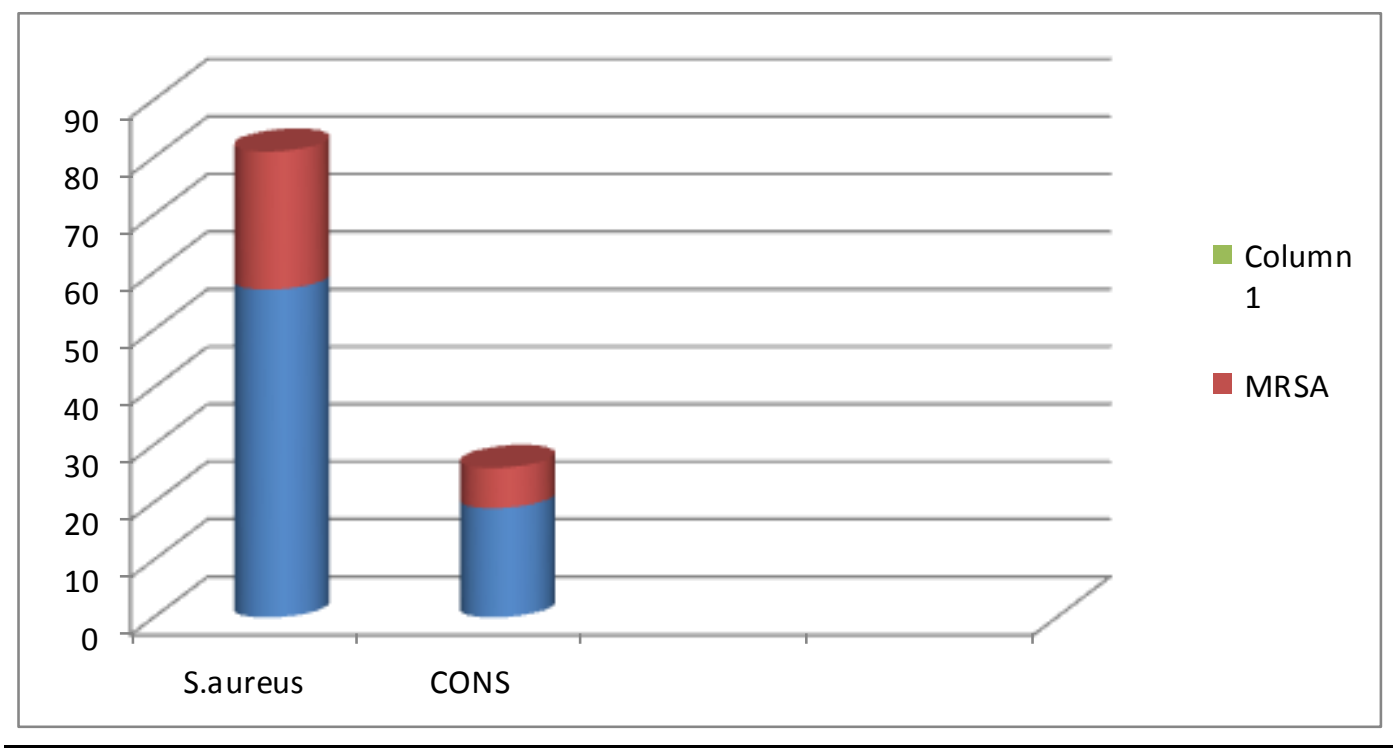

\section{Implications}

Early diagnosis ensures that appropriate measures can be taken to limit the spread of the infection. If CA-MRSA is detected early it can be treated effectively with appropriate antibiotics. In order to prevent the spread of infections, it is important to recommend the health care professionals to practise Standard Precaution like hand hygiene, aseptic precautions, clean environment, barrier precautions. Decolonisation of patient to be considered in case of recurrent infections and ongoing transmission among close contacts. Educating the patient on appropriate wound care, personel hygiene and not sharing personel items, maintaining clean environment to prevent the spread of MRSA and outbreaks.

\section{References}

1. Hay RJ, Adriaans BM. Bacterial infections. In: Burns T, Breathnach S, Cox X, Griffiths C, editors. Rook's Textbook of Dermatology. 7th ed. Vol. 2. Oxford: Blackwell Science Ltd; 2004. pp. 27.1-85.
2. Paniker CK. Staphylococcus. In: Ananthanarayan R, Paniker CK, editors. Ananthanarayan and Paniker's Textbook of Microbiology. 7th ed. Hyderabad: Orient Longman Private Limited; 2005. pp. 192-201.

3. Grundmann H, Aries-de-Sousa M, Boyce J, Tiemersma E. Emergence and resurgence of methicillinresistance Staphylococcus aureus as a public-health threat. Lancet. 2006; 368: 874-85.

4. Salgado CD, Farr BM, Calfee DP. Community-acquired methicillinresistance Staphylococcus aureus: A meta-analysis of prevalence and risk factors. Clin Infect Dis. 2003; 36: 1319.

5. Occurrence and Antimicrobial Susceptibility Pattern of Community and Hospital associated Methicillin resistant Staphylococcus aureus strains in Sikkim. O. Kunsang Bhutia, T.S.K. Singh Department of Microbiology, Sikkim Manipal Institute of Medical Sciences, East Sikkim, India. JIMSA OctoberDecember 2012 Vol. 25 No.4 


\section{How to cite this article:}

Neelaveni Duraisamy, Nirmala Devi Somasundram and Vidhyarani Rangasamy. 2020. Community Acquired Methicillin Resistant Staphylococcus aureus (CA-MRSA) among Skin and Soft Tissue Infections. Int.J.Curr.Microbiol.App.Sci. 9(02): 652-657. doi: https://doi.org/10.20546/ijcmas.2020.902.080 